\title{
Design controversies and the generation effect: Support for an item-order hypothesis
}

\author{
MATT SERRA and JAMES S. NAIRNE \\ Purdue University, West Lafayette, Indiana
}

\begin{abstract}
We performed three experiments to investigate an earlier finding of Nairne, Riegler, and Serra (1991) that item generation disrupts the long-term retention of serial order. Experiment 1 demonstrated a clear advantage of reading over generating on a reconstruction test when reading and generating occurred in pure, but not mixed, lists. Experiment 2 showed that the standard generate advantage is seen in free recall of mixed, but not pure, lists, even when recall is im. mediately followed by reconstruction of serial order of the same items. Experiment 3 replicated Experiment 1, but with the use of an incidental learning procedure. The results of all three experiments are consistent with the claim that generation has dissociative effects on item and order memory; moreover, these dissociative effects help to explain design controversies in the generation effect literature.
\end{abstract}

Among the recent controversies in the generation effect literature is the finding that the mnemonic benefits of generation may depend on the nature of the experimental design. Typically, the advantage of generation over reading is more robust in a within-list design than in a between-list design (Slamecka \& Katsaiti, 1987). Furthermore, its expression in either of these design types depends on the memory test used. In the case of recognition, the generation effect seems to occur regardless of design (Begg \& Snider, 1987; Begg, Snider, Foley, \& Goddard, 1989; Graf, 1980; Hirshman \& Bjork, 1988; Nairne, Riegler, \& Serra, 1991; Slamecka \& Graf, 1978; Slamecka \& Katsaiti, 1987; Watkins \& Sechler, 1988). When free recall is used as the memory test, however, the generation of items typically produces enhancement only when read and generated items are mixed in the same list (Begg \& Snider, 1987; Hirshman \& Bjork, 1988; McDaniel, Waddill, \& Einstein, 1988; Slamecka \& Katsaiti, 1987). In some instances, a distinct advantage of reading over generation (negative generation effect) has been shown when free recall is used in a between-list design (Nairne et al., 1991; Schmidt \& Cherry, 1989).

Several explanations have been given for the withinversus between-list findings. Most of these accounts are based on the idea that generation has enhancing effects on item and/or relational information (Begg \& Snider, 1987; Begg et al., 1989; Hirshman \& Bjork, 1988; McDaniel \& Waddill, 1990; McDaniel et al., 1988; Schmidt \& Cherry, 1989; Slamecka \& Katsaiti, 1987). For exam-

We thank N. Slamecka, E. Hirshman, J. Gardiner, and the editor for their critical comments on an earlier version of the manuscript. Thanks are also due to Cynthia Aguilar, Nicole Antonelli, Alberder Hampton, Karen Hoyne, Christine Mentzer, Andy Robinson, and Karen Whelan for help in conducting these experiments. Correspondence can be addressed to either author at the Psychology Department, Purdue University, West Lafayette, IN 47907-1364. ple, Begg et al. (1989) propose that generation, through enhancement of item-specific features, creates a distinctive candidate for the subject to use at the time of test. Items are distinctive only in relation to some background, however, and when all items receive the same encoding manipulation (e.g., generation), enhanced item-specific features become less effective retrieval cues. Item enhancement views have been successful when recognition is used as the dependent variable, but they have trouble with studies demonstrating that generation affects relational information (e.g., cue-target associations) as well as item-specific information (cf. McDaniel \& Waddill, 1990). In contrast, multifactor theories (Hirshman \& Bjork, 1988; McDaniel et al., 1988) propose that both item and relational information are enhanced by the generation of items. Generation can serve to increase activation of the features of the response item, cue-target associative relations, and even whole-list factors. The multifactor explanations have had some success in explaining design effects, but specification of exactly what is meant by the idea of relational information, and how it is measured, varies from article to article.

We are mainly concerned with a recent explanation proposed by Nairne et al. (1991) that rests on the historical distinction between item and order information (e.g., Healy, 1974; Murdock, 1974). Both types of mnemonic information contribute significantly to memory performance; in fact, the relative contributions of each can importantly determine the particular performance pattern that is obtained (for a review, see Murdock, 1974). In the Nairne et al. experiments, long-term retention of serial order was assessed either in a reconstruction task or in terms of the amount of input-output correspondence in free recall. The results showed that when subjects were required to complete word fragments at the time of presentation, long-term retention of serial order was significantly impaired. However, memory for the individual 
items, assessed through recognition, was helped by the generation task. The disruption of order information, it was argued, typically masks the benefit gained from improved item information in between-list designs because subjects often rely on serial order as an output strategy in free recall (see, e.g., Postman, 1972). For mixed lists, where items to be read or generated are randomly placed in the same list, generation was assumed to disrupt the order-based recall strategy equally for both item types, unmasking the item-based generation advantage.

Nairne et al. (1991) used only between-list designs in their experiments; consequently, that research provided no direct evidence to support our claim about mixed lists. Accordingly, we designed Experiment 1 to test whether item generation disrupts memory for order in mixed, as well as pure, lists. In contrast to the predictions for the pure lists, for mixed lists our view predicts that order information will be disrupted equally for read and generated items. This logic is based on the idea that disruption of any given serial position (e.g., the third item on the list) will spill over and affect the retention of neighboring serial positions (i.e., the second and fourth serial positions). Experiment 2 replicated Experiment 1, but also included a free recall test preceding each reconstruction test. For free recall, we expected to replicate previous findings of no positive generation effect for pure lists, but a positive generation effect for the mixed lists. Finally, in Experiment 3, we used an incidental learning paradigm in an attempt to replicate the Experiment 1 findings. The generation effect is commonly found in incidental learning (see, e.g., Watkins \& Sechler, 1988), and it is important to show that our claims about the disruption of order information apply even when the subject is not expecting an order test.

\section{EXPERIMENT 1}

In our first experiment, subjects saw three types of stimulus lists. The lists contained (1) all complete items (read lists), (2) all fragmented items (generate lists), or (3) both complete and fragmented items (mixed lists). Because we were primarily interested in the effect of generation on memory for order, a reconstruction task was used as the test of memory. The reconstruction task is designed to measure memory for order information alone, by supplying the subject with all of the item information necessary to complete the task. Following Nairne et al. (1991), better reconstruction performance was expected for the pure read lists than for the pure generate lists. For the mixed lists, we reasoned that disrupting order information for the generate items would spill over to the read items; we anticipated no difference in reconstruction performance for read and generate items within the mixed lists, but somewhat lower performance overall than that for the pure read lists.

\footnotetext{
Method

Subjects and Apparatus. A total of 46 subjects ( 26 females and 20 males) participated for extra credit in an introductory psychol-
}

ogy course. The subjects were tested individually, with each session lasting approximately $1 \mathrm{~h}$. The presentation of stimulus materials was controlled by an IBM-compatible computer. The subjects wrote their responses on answer sheets provided by the experimenter.

Materials. The stimulus items were 192 nouns, four to six letters in length. All items were drawn from the Paivio, Yuille, and Madigan (1968) norms. The items were medium- to high-frequency nouns, with medium to high imagery (5.58), concreteness (5.59), and meaningfulness (6.37) values. To create the fragment form of each item, one letter was replaced with a single underscore. Two criteria were used in the creation of these fragmented items. First, to minimize incorrect fragment completions (generation failures), the solution was designed to be as obvious as possible. Second, the fragment presented could be completed in only one way. For example, the solution to the fragment HOU__ would be HOUR. The 192 items were placed into 24 unique eight-item lists with each item randomly assigned to one of the eight serial positions. Across subjects, all items appeared in either complete or fragment form, and the list sequences were completely counterbalanced across the conditions of interest. This was accomplished by first generating a random ordering of the three list types. Then new orderings were created by changing read lists to mixed lists, generate lists to read lists, mixed lists to generate lists, and so on. Within the mixed lists, read and generate items occurred equally often at each of the eight serial positions.

Procedure. Each session consisted of 3 eight-item practice lists (generate, read, and mixed) and the 24 eight-item test lists. The 24 test lists were made up of 8 read lists, 8 generate lists, and 8 mixed lists, randomly presented. Each list was followed by a distractor task and then a reconstruction task. All subjects were made aware of the requirements of the reconstruction task before beginning the experiment.

Each trial began with the word "READY," accompanied by a short tone. The eight list items were then presented, one at a time, in the center of the computer screen. Each remained on the screen for $2 \mathrm{sec}$ with a .5-sec interstimulus interval. The items appeared in either a complete or a fragmented form, and the subjects were required to say each item aloud, completing a fragment when necessary. Any incorrect completions (generation failures) were recorded by the experimenter. After the last item for a given list appeared, the subjects completed a 30-sec digit tracking distractor task. The distractor task required subjects to press one key if a digit that appeared in the center of the screen was even, and another key if the digit was odd. The digits were selected randomly from the set 1-9 and appeared on the screen at a rate of one per second. After the completion of the distractor task, the subjects were required to perform the reconstruction task for that list.

At the time of the reconstruction task, all eight items from that trial were presented at the same time across the center of the screen, but in a new random order. The subjects were required to write the eight items on their answer sheet in the original order of presentation. The answer sheet contained 24 rows of eight blank spaces each. The subjects were instructed to fill in each of the eight blank spaces and to use each item only once. The reconstruction task was subject paced. The eight items remained on the screen until the space bar was pressed, which initiated the next trial.

\section{Results and Discussion}

There were no read errors and subjects correctly generated the fragmented item on $93.8 \%$ of the opportunities. The patterns of results did not vary when the analyses were performed on data conditionalized on success or failure to generate items. Therefore, only analyses on unconditionalized data are presented. All effects reported as significant are so at a minimum $p<.05$ level. 
Table 1

Reconstruction Performance on the Pure and Mixed Lists in Experiment 1, Presented as a Function of Serial Position

\begin{tabular}{|c|c|c|c|c|c|c|c|c|c|}
\hline \multirow[b]{2}{*}{ List Type } & \multicolumn{8}{|c|}{ Serial Position } & \multirow[b]{2}{*}{$M$} \\
\hline & 1 & 2 & 3 & 4 & 5 & 6 & 7 & 8 & \\
\hline Pure read & .88 & .75 & .67 & .62 & .61 & .57 & .62 & .73 & .68 \\
\hline Pure generate & .83 & .70 & .55 & .47 & .45 & .47 & .51 & .66 & .58 \\
\hline Mixed read & .90 & .74 & .59 & .52 & .47 & .55 & .57 & .69 & .63 \\
\hline Mixed generate & .84 & .72 & .60 & .57 & .54 & .51 & .56 & .69 & .63 \\
\hline
\end{tabular}

The overall results of the reconstruction test are shown in Table 1. This table presents mean proportion correct reconstruction, by serial position, for the various conditions. These data show how well subjects were able to place the items back into their original order of presentation, broken down for each position in a list. An overall analysis of variance (ANOVA), collapsed across read and generate items in the mixed lists, was performed on the number of correct item placements for each serial position. There were significant main effects of serial position $\left[F(7,315)=50.92, M S_{\mathrm{e}}=.170\right]$ and list type $[F(2,90)=$ 13.11, $\left.M S_{\mathrm{e}}=.024\right]$. The list type $\times$ serial position interaction failed to reach significance $[F(14,315)=1.20$, $p \leq .268$ ].

Follow-up comparisons, using the Dunn correction procedure (see Keppel, 1973), confirmed that reconstruction performance on the pure read list was significantly better than performance on the pure generate list $[F(1,90)=$ $\left.26.19, M S_{\mathrm{e}}=.024\right]$. Of the 46 subjects in this experiment, 35 showed better reconstruction performance on the read lists, 10 showed better performance on the generate lists, and there was 1 tie. These results replicate the Nairne et al. (1991, Experiment 1) findings. Overall performance on the mixed list fell between that of the read and generate lists: Performance on the mixed list was significantly lower than that on the pure read list $[F(1,90)=$ $\left.7.17, M S_{\mathrm{e}}=.007\right]$, but was significantly better than that on the pure generate list $\left[F(1,90)=5.96, M S_{\mathrm{e}}=.005\right]$. This would be the expected result if the negative effects of generating a subset of the list items spilled over to the read items, disrupting order retention of the list as a whole.

The reconstruction data for the read and generate items within the mixed lists are shown in the bottom two rows of Table 1 . Because both read and generate items appeared equally often at each serial position across lists, the mixed trials could be broken down into read and generate items. An ANOVA was then performed on correct reconstruction performance by serial position. The analysis revealed a significant main effect of serial position $[F(7,315)=$ $\left.29.42, M S_{\mathrm{e}}=.140\right]$, but neither item type $(\mathrm{F}<1)$ nor the item type $\times$ serial position interaction $[F(7,315)=$ $\left.1.01, M S_{\mathrm{e}}=.427\right]$ was significant in the analysis. Therefore, reconstruction performance was not differently affected by whether the item was read or generated. Of the 46 subjects, 19 showed better reconstruction performance on the read items, and 20 showed better performance on the generate items; there were 7 ties. These results support the assertion of Nairne et al. (1991) that generation of a random half of the items within a list disrupts order memory equally for all the items in the list.

The demonstration of a null effect of the read versus generate variable on reconstruction performance within a mixed list is an important first step in testing the itemorder hypothesis. However, it is important to demonstrate the more typical finding of a generation effect in the free recall of mixed lists (see, e.g., Begg \& Snider, 1987; Hirshman \& Bjork, 1988) under conditions in which subjects are expecting an order test. According to Nairne et al. (1991), one typically sees a generate advantage in the free recall of mixed lists because memory for the generated items is enhanced but not masked by a differential disruption of order information. To that end, we designed Experiment 2 to assess free recall performance, as well as to replicate the results of Experiment 1 .

\section{EXPERIMENT 2}

In Experiment 2, subjects again saw read, generate, and mixed lists. On each trial, the subjects were required first to recall each list and then immediately to reconstruct the serial order. The free recall task was designed to determine whether a positive generation effect would occur if subjects were also expecting an order test, at least for the mixed lists. In the pure list case, no positive generation effect was expected, given the results of Nairne et al. (1991). Performance on the reconstruction task was predicted to replicate that in Experiment 1 . In addition, because subjects both recalled and reconstructed items on every trial, it was possible to examine recall performance conditionalized on correct reconstruction.

\section{Method}

Subjects and Apparatus. A total of 24 subjects (11 males and 13 females) participated for extra credit in an introductory psychology course. The subjects were tested individually, with each session lasting approximately $1 \mathrm{~h}$. The presentation of stimulus materials was controlled by an IBM-compatible computer. The subjects wrote their responses on answer sheets provided by the experimenter.

Materials and Procedure. No changes were made in stimulus materials. The major change between Experiments 1 and 2 was that on each trial, the subjects were required to perform a free recall task prior to reconstructing the order of each list. After the presentation of the last digit of the distractor task, the message "RECALL NOW" appeared on the computer screen. The subjects were told to write down as many of the items as they could remember in any order. The subjects wrote their responses on a separate page of a response booklet for each list. Following free recall, the subjects were asked to turn the page of the booklet and press the space bar to begin the reconstruction task. The reconstruction procedure was identical to the one used in Experiment 1. The eight items from each list were presented again on the computer screen, in a new random order, and the subjects wrote them on their answer sheets in the original order of presentation. There were three practice trials. Both the free recall and the reconstruction tasks were subject paced.

\section{Results and Discussion}

The subjects successfully generated the fragmented items on $92.9 \%$ of the generation opportunities and were $99.7 \%$ correct on the read items. 
Table 2

Mean Proportion Correct Recall on the Read and Generate Items in Pure and Mixed Lists in Experiment 2, Presented as a Function of Serial Position (Conditionalized on Correct Generation) Serial Position

\begin{tabular}{|c|c|c|c|c|c|c|c|c|c|}
\hline List Type & 1 & 2 & 3 & 4 & 5 & 6 & 7 & 8 & $M$ \\
\hline Pure read & .75 & .64 & .59 & .49 & .53 & .61 & .62 & .75 & .62 \\
\hline Pure generate & .54 & .51 & .48 & .45 & .45 & .54 & .66 & .75 & .55 \\
\hline Mixed read & .68 & .49 & .41 & .45 & .38 & .54 & .58 & .66 & .52 \\
\hline Mixed generate & .67 & .55 & .51 & .35 & .62 & .54 & .66 & .72 & .58 \\
\hline
\end{tabular}

Free recall. Table 2 shows mean proportion correct recall for the read and generate items in the pure and mixed lists as a function of serial position; these data are conditionalized on correct generation because, unlike in the reconstruction test, items were not presented again at the point of recall. Mean performance collapsed across serial position is seen in the far right column. An overall ANOVA, collapsed across read and generated items in the mixed lists, revealed significant effects of serial position $\left[F(7,161)=17.59, M S_{\mathrm{e}}=.036\right]$, list type $[F(2,46)=$ $\left.10.23, M S_{\mathrm{e}}=.034\right]$, and the list type $\times$ serial position interaction $\left[F(14,322)=1.78, M S_{e}=.027\right]$. A similar analysis on the unconditionalized data produced the same pattern, except that the list type $\times$ serial position interaction was not significant in this analysis.

Subsequent comparisons indicated that recall performance on the pure read lists was significantly better than on the pure generate lists $\left[F(1,46)=15.64, M S_{e}=.034\right]$ or the mixed lists $\left[F(1,46)=15.05, M S_{\mathrm{e}}=.034\right]$. To the extent that memory for serial order is an important determinant of free recall, because subjects often employ an order-based output strategy, better recall for the pure read lists is a reasonable result. Negative generation effects of this type have been obtained before (e.g., by Nairne et al., 1991; Schmidt \& Cherry, 1989), and their occurrence presumably results from an impaired output strategy brought about by the negative effect that generation has on memory for serial order. The performance difference between pure generate and mixed lists was not significant $[F(1,46)<1]$.

The bottom two rows of Table 2 show the mean proportion correct recall of read and generate items within the mixed list. Once again, because both read and generate items occurred equally often at each serial position, we were able to break the results down into proportion correct for each item type at each serial position. The ANOVA revealed significant main effects for item type $\left[F(1,23)=5.82, M S_{\mathrm{e}}=.053\right]$ and serial position $\left[F(7,161)=7.26, M S_{\mathrm{e}}=.070\right]$. The item type $\times$ serial position interaction did not reach significance $[F(7,161)=$ $1.99, p \leq .06$ ]. Of the 24 subjects, 16 recalled generate items better and 8 recalled read items better. An additional analysis of the unconditionalized data produced the same results. The demonstration of a positive generation effect when items from mixed lists were recalled, along with the opposite result in the pure list design, replicated the standard findings in the literature (e.g., Slamecka \& Katsaiti, 1987).

Reconstruction. The results of the reconstruction task are shown in Table 3. Despite the fact that reconstruction followed recall on each trial, the results still resembled those seen in Experiment 1. The ANOVA revealed significant main effects of serial position $[F(7,161)=$ $\left.79.61, M S_{\mathrm{e}}=.170\right]$ and list type $[F(2,46)=12.56$, $\left.M S_{\mathrm{e}}=.024\right]$. The list type $\times$ serial position interaction failed to reach significance $[F(14,322)=1.09, p \leq$ .362]. Follow-up comparisons confirmed that reconstruction performance on the pure read list was significantly better than performance on the pure generate list $\left[F(1,46)=7.97, M S_{e}=.007\right]$, but in Experiment 2 , the read-mixed difference was not significant $[F(1,46)<1]$. Of the 24 subjects in this experiment, 18 showed better reconstruction performance on the read lists and 6 showed the opposite pattern. The reconstruction data for the read and generate items within the mixed list are shown in the bottom two rows of Table 3. As in Experiment 1, the ANOVA revealed a significant main effect of serial position $\left[F(7,161)=24.38, M S_{\mathrm{e}}=.140\right]$, but no effect of item type $(F<1)$. The act of fragment completion, compared with reading, produced no differential effect on reconstruction performance. Of the 24 subjects, 14 showed better performance on the read items and 10 showed better performance on the generate items. These results replicated the null effect of item type on reconstruction performance in the mixed list seen in Experiment 1.

Conditionalized recall. As mentioned earlier, the design of Experiment 2 afforded the opportunity to look at recall performance conditionalized on reconstruction performance. Collapsed across all list types, there was a strong positive correlation between recall performance and reconstruction performance (Pearson $r=.85$ ). If a disruption in memory for serial order typically masks itembased enhancements from generation, then larger positive generation effects should emerge on trials where subjects retain good memory for order. To check on this prediction, we examined recall performance for read and generated items only on trials in which subjects showed perfect reconstruction of order. For mixed lists, the size of the generation effect was clearly enhanced relative to the overall data (mean performance of .82 and .69 for the generate and read items, respectively). In the pure lists, although a positive generation effect was not obtained, the negative generation effect seen in the overall data was elimi-

Table 3

Reconstruction Performance on the Read and Generate Items in the Pure and Mixed Lists in Experiment 2, Presented as a Function of Serial Position

\begin{tabular}{lccccccccc}
\hline & \multicolumn{8}{c}{ Serial Position } \\
\cline { 2 - 9 } \multicolumn{1}{c}{ List Type } & 1 & 2 & 3 & 4 & 5 & 6 & 7 & 8 & $M$ \\
\hline Pure read & .82 & .70 & .56 & .42 & .38 & .34 & .41 & .46 & .52 \\
Pure generate & .80 & .70 & .49 & .33 & .26 & .28 & .31 & .45 & .45 \\
Mixed read & .79 & .73 & .48 & .46 & .32 & .37 & .30 & .54 & .50 \\
Mixed generate & .88 & .64 & .52 & .37 & .38 & .35 & .38 & .44 & .50 \\
\hline
\end{tabular}


nated (mean performance of .80 and .81 for the generate and read items, respectively). Thus, for both the pure and the mixed lists, improved order memory mapped onto better generate relative to read performance, as predicted by the item-order hypothesis of Nairne et al. (1991).

The results of Experiments 1 and 2 are consistent with the item-order hypothesis proposed by Nairne et al. (1991). However, one might still argue that the negative effect of generation on order could be attributed to the task demands anticipated by the subjects. In both the Nairne et al. study and Experiments 1 and 2 of the present report, subjects were expecting an order test. This expectancy might have led them to pay more attention to order information than would normally be the case. To address this issue, in Experiment 3 we measured order retention in an incidental learning task.

\section{EXPERIMENT 3}

In this experiment, the subjects were presented with three 8-item lists of words and were instructed to give pleasantness ratings to each word as it was presented. Everyone received one pure generate list, one pure read list, and one mixed list containing 4 fragmented and 4 intact items. After the subjects had made pleasantness ratings for each of the 24 items, they performed a distractor task. At the completion of the distractor task, everyone was given a surprise reconstruction task for the three lists. If the item-order explanation held, two findings should emerge. First, the reconstruction performance on the pure read lists (complete items) should be superior to that on the pure generate (all fragmented) lists. Second, there should be no significant difference in reconstruction performance for the read and generate items within the mixed list.

\section{Method}

Subjects and Apparatus. A total of 96 subjects (51 females and 45 males) participated in this experiment. All subjects received extra credit in introductory psychology for their participation. The subjects were tested in groups of 4 to 8 , with each session lasting approximately $30 \mathrm{~min}$. The stimulus materials were presented with a Kodak Ectographic Model 591526 slide projector. The timing of the stimulus presentation was controlled by a Kodak EC Model III automatic timer. The distractor task was timed with a hand-held digital stopwatch. The subjects wrote their responses on answer sheets provided by the experimenter.

Materials. The stimulus items were 24 nouns, drawn from the pool of 192 items used in Experiments 1 and 2. Because a group procedure was used in Experiment 3, confidential monitoring of generation failures was not possible. Consequently, only items that resulted in zero generation failures in Experiments 1 and 2 were used. The 24 items were placed into three unique 8-item lists with each item randomly assigned to one of the eight serial positions. All items appeared in either complete or fragment form within their respective lists, and the lists were completely counterbalanced across the conditions of interest. That is, each list appeared in a read (all complete items), generate (all fragmented items), and mixed (four read and four fragmented items) form across groups of subjects. Within the mixed list, both read and generate items appeared equally often in each serial position across subjects.
Procedure. Each session began with the experimenter reading a set of instructions describing the rating task. The subjects were told that they would be rating the pleasantness of a set of words. The set of words was made up of three lists of eight words; each word was presented one at a time in the center of the projection screen. As each word appeared on the projection screen, the subjects rated the word on a scale from 1 (unpleasant) to 3 (pleasant). The subjects were also instructed that some of the words would be presented in incomplete or fragment form. When these words appeared on the screen, the subjects were asked to complete the fragmented words to themselves prior to making their ratings. The ratings were made on a response sheet that had the rating scale at the top, a one-line instruction, and then three numbered rows of eight blanks. The subjects wrote the number corresponding to the pleasantness $(1-3)$ of the word on the screen in the corresponding blank (1-8) on the rating sheet. Each word was presented for $2 \mathrm{sec}$. There was a 6-sec pause between the last word of a list and the first word of the next list.

After the subjects had rated the last word of the third list, they were instructed to turn their rating sheet over and begin counting backwards by ones from 100 on the rating sheet until told to stop. The distractor task lasted $1 \mathrm{~min}$. The rating sheets were then collected and the surprise reconstruction task was distributed. The reconstruction task consisted of a response sheet containing the three lists of words each in a new random order. Underneath each of the lists of eight words was a list of eight blank lines. The subjects were instructed to place the words from each list in their original order of presentation by writing the words in the blanks immediately below each list. The subjects were instructed to use each word only once and to put a line through each word as it was used. The reconstruction task was subject paced.

\section{Results and Discussion}

The overall results of the reconstruction test can be seen in Table 4. As in Experiment 1 , a $3 \times 8$ within-subject ANOVA was performed on the number of correct item placements for each serial position. The ANOVA revealed significant main effects of serial position $[F(7,665)=$ $\left.48.45, M S_{\mathrm{e}}=.182\right]$ and list type $[F(2,190)=11.99$, $\left.M S_{\mathrm{e}}=.304\right]$; the list type $\times$ serial position interaction failed to reach significance $\left[F(14,1,330)=1.33, M S_{\mathrm{e}}=\right.$ .180]. As expected, planned comparison analyses confirmed that reconstruction performance on the pure read list was significantly better than performance on the pure generate list $\left[F(1,95)=18.80, M S_{\mathrm{e}}=.312\right]$. Performance was significantly lower on the mixed list than on the read list $\left[F(1,190)=16.98, M S_{\mathrm{e}}=.304\right]$, but it did not differ from that on the generate list $[F(1,190)<1]$. As in Experiment 1, the majority of the subjects in this experiment showed better reconstruction performance on

Table 4

Reconstruction Performance on the Read and Generate Items in the Pure and Mixed Lists in Experiment 3, Presented as a Function of Serial Position

\begin{tabular}{lccccccccc}
\hline & \multicolumn{8}{c}{ Serial Position } \\
\cline { 2 - 9 } \multicolumn{1}{c}{ List Type } & $\mathbf{1}$ & $\mathbf{2}$ & $\mathbf{3}$ & $\mathbf{4}$ & $\mathbf{5}$ & $\mathbf{6}$ & $\mathbf{7}$ & $\mathbf{8}$ & $\boldsymbol{M}$ \\
\hline Pure read & .82 & .57 & .39 & .33 & .27 & .32 & .32 & .53 & .44 \\
Pure generate & .67 & .33 & .17 & .24 & .18 & .25 & .33 & .41 & .32 \\
Mixed read & .78 & .44 & .22 & .20 & .20 & .20 & .30 & .30 & .32 \\
Mixed generate & .72 & .42 & .26 & .14 & .22 & .22 & .26 & .32 & .33 \\
\hline
\end{tabular}


the read lists than on the generate lists $(60$ read, 25 generate, and 11 ties).

Reconstruction performance for the read and generate items within the mixed list can be seen in the bottom two rows of Table 4 . As in the previous two experiments, a $2 \times 8$ ANOVA was performed on correct reconstruction performance $\times$ serial position for the read and generate items. There was a significant main effect of serial position $\left[F(7,665)=19.30, M S_{\mathrm{e}}=.087\right]$, but neither item type $(F<1)$ nor the item type $\times$ serial position interaction $(F<1)$ was reliable in the analysis. Of the 96 subjects, 30 showed better reconstruction performance on the read items, 29 showed better reconstruction performance on the generate items, and there were 37 ties.

In this experiment, we replicated the main pure and mixed-list reconstruction results of Experiments 1 and 2 by using an incidental rather than an intentional learning task. The results therefore argue against a simple-minded task demand explanation of the experimental results in Nairne et al. (1991) and in the previous two experiments. It appears that the disruption of order information from generation is a general phenomenon that is not restricted to situations in which subjects are expecting an order test. Moreover, we again found no evidence of a read-generate difference for items within a mixed list. With this result, we replicated the null effect of item type (read vs. generate) on reconstruction in the within-list case seen under the intentional learning demands of Experiments 1 and 2.

\section{GENERAL DISCUSSION}

In the three experiments presented here, we have provided further evidence for the dissociative effect that item generation can have on memory for item and order information. In Experiment 1, we replicated earlier findings obtained with lists containing only read or generated items, and we extended the analysis to mixed lists. In Experiment 2, we were able to show that generation can lead to substantial mnemonic benefits under conditions in which no differential disruption of order information has occurred (i.e., in mixed lists). In Experiment 3, we addressed a possible alternative explanation for the results seen here and in Nairne et al. (1991)-subject expectancies based on demands of the memory task-by replicating the general data patterns in an incidental learning environment.

Of main interest is the relevance of these dissociative effects to current design controversies in the generation effect literature. It is often the case that no generation advantage is seen when reading and generating occur in different lists and free recall is used as the retention measure (Begg \& Snider, 1987; Graf, 1980; Hirshman \& Bjork, 1988; McDaniel et al., 1988; Nairne et al., 1991; Slamecka \& Graf, 1978; Slamecka \& Katsaiti, 1987). For a within-list design, in contrast, free recall typically produces a positive generation effect (Begg \& Snider, 1987; Begg et al., 1989; Hirshman \& Bjork, 1988; Slamecka \& Katsaiti, 1987; Watkins \& Sechler, 1988). According to Nairne et al. (1991), when all of the items within a list have been generated, enhanced item information produced by generation can be masked in recall because subjects often rely on an order-based retrieval strategy. That is, there is a tradeoff involved in the recall of pure generate lists: Recall is enhanced by the improved item information, but subjects are unable to make full use of this information because of an impaired output strategy. The items in the read lists receive no benefit in item memory from generation, but also receive no impairment of order memory. If a retrieval strategy other than one based on serial order is available (e.g., when categorized lists are used), the benefits of generation are unmasked. This unmasking results in a generation effect in free recall (McDaniel et al., 1988; Nairne et al., 1991).

For mixed lists, where both intact and fragmented items are randomly combined in the same list, generation appears to affect the retention of serial order for all items, regardless of whether they have been generated or read. This overall disruption allows any item enhancement that those generated items may have obtained to come to the surface, resulting in the within-list positive generation effect for free recall. The addition of the mixed-list groups in the experiments reported here allowed for a test of this assumption. The fact that order was disrupted for items in the mixed list, relative to the pure read condition, but not differentially for the read and generated items, provides support for the overall account of Nairne et al. (1991).

Two theoretical issues remain unresolved: First, why is order information disrupted by generation? One possibility is that the time taken to generate is taxing, leaving less time for the subject to attend to any order information. The fact that order is disrupted in an incidental learning context as well, however, suggests that the locus of the disruption is not strategic. Clearly, reading and generating require the subject to engage in different kinds of encoding operations and to attend to different stimulus elements. It seems likely that generation induces item-specific processing that simply does not transfer well to a task requiring knowledge about how items are related along a temporal dimension. Second, why is order information disrupted equally for both item types in a mixed list? Presumably the retention of any given item's serial position is affected by the corresponding retention of its neighbors'. If the subject's memory for what occurred in the third serial position is impaired because that item was generated, then less information is available to ascertain where the remaining items in the list may have occurred.

We believe that studies that increase our understanding of the generation effect can play an important role in our understanding of mnemonic phenomena in general. The use of this robust phenomenon as an investigative tool is becoming more and more widespread. For example, the generation effect has been used in the study of person perception and person memory (Lingle, Geva, Ostrom, Leippe, \& Baumgardner, 1979). The generation effect has also been used in the study of expert/novice differences 
(Reardon, Durso, Foley, \& McGahan, 1987) and in the study of social inference and memory (Carlston, 1980). More recently, the debate between data-driven and conceptually driven processes (Gardiner, 1989; Jacoby, 1983; Roediger \& Blaxton, 1987; Roediger \& Weldon, 1987) as opposed to a memory systems approach to memory in general (Gardiner, 1989) has made much use of the generation phenomenon. A deeper understanding of the phenomenon itself is clearly needed if this type of use is to continue to be as fruitful as it has been in the past.

\section{REFERENCES}

BEGG, I., \& SNider, A. (1987). The generation effect: Evidence for generalized inhibition. Journal of Experimental Psychology: Leaming. Memory, \& Cognition, 13, 553-563.

BEGG, I., Snider, A., Foley, F., \& Goddard, R. (1989). The generation effect is no artifact: Generation makes words distinctive. Journal of Experimental Psychology: Learning, Memory, \& Cognition, 15, 977-989.

CARLSTON, D. E. (1980). The recall and use of traits and events in social inference processes. Journal of Experimental Social Psychology, 16, 303-328.

GARDINER, J. M. (1989). A generation effect in memory without awareness. British Journal of Psychology, 80, 163-168.

GRAF, P. (1980). Two consequences of generating: Increased inter- and intraword organization of sentences. Journal of Verbal Learning \& Verbal Behavior, 19, 316-327.

Healy, A. F. (1974). Separating item from order information in shortterm memory. Journal of Verbal Learning \& Verbal Behavior, 13 644-655.

Hirshman, E., \& Bjork, R. A. (1988). The generation effect: Support for a two factor theory. Journal of Experimental Psychology: Learning, Memory, \& Cognition, 14, 484-494.

JACOBY, L. L. (1983). Remembering the data: Analyzing interactive processes in reading. Journal of Verbal Learning \& Verbal Behavior, 22, 485-508

KePPEL, G. (1973). Design and analysis. Englewood Cliffs, NJ Prentice-Hall

Lingle, J. H., Geva, N., Ostrom, T. M., Leippe, M. R., \& BaumGARDNER, M. H. (1979). Thematic effects of person judgments on impression organization. Journal of Personality \& Social Psychology, 37, 674-687

McDaniel, M. A. \& Waddill, P. J. (1990). Generation effects for context words: Implications for item-specific and multifactor theories. Journal of Memory \& Language, 29, 201-211.

McDaniel, M. A., Waddill, P. J., \& Einstein, G. O. (1988). A contextual account of the generation effect: A three factor theory. Journal of Memory \& Language, 27, 521-536.

Murdock, B. B., JR. (1974). Human memory: Theory and data. Hillsdale, NJ: Erlbaum.

Nairne, J. S., Riegler, G. J., \& Serra, M. (1991). Dissociative effects of generation on item and order information. Journal of Experimental Psychology: Learning, Memory, \& Cognition, 17, 702-709.

Paivio, A., Yuille, J. C., \& Madigan, S. A. (1968). Concreteness, imagery, and meaningfulness values for 925 nouns. Journal of Experimental Psychology Monographs, 76(1, Pt. 2).

Postman, L. (1972). A pragmatic view of organization theory. In E. Tulving \& W. Donaldson (Eds.), Organization and memory (pp. 3-48). San Diego, CA: Academic Press.

Reardon, R., Durso, F. T., Foley, M. A., McGahan, J. R. (1987). Expertise and the generation effect. Social Cognition, 5, 336-348

RoEdiger, H. L., III, \& Blaxton, T. A. (1987). Retrieval modes produce dissociations in memory for surface information. In D. Gorfein \& R. R. Hoffman (Eds.), Memory and cognitive processes: The Ebbinghaus centennial conference (pp. 349-379). Hillsdale, NJ: Erlbaum.

Roediger, H. L., III, WeLDON, M. S. (1987). Reversing the picture superiority effect. In M. A. McDaniel \& M. Pressley (Eds.), Imagery and related mnemonic processes: Theories, individual differences, and applications (pp. 151-174). New York: Springer-Verlag.

SCHMidT, S. R. \& CHERRY, K. (1989). The negative generation effect: Delineation of a phenomenon. Memory \& Cognition, 17, 359-369.

Slamecka, N. J., \& GRAF, P. (1978). The generation effect: Delineation of a phenomenon. Journal of Experimental Psychology: Learning, Memory, \& Cognition, 4, 592-604.

Slamecka, N. J., \& Katsaiti, L. T. (1987). The generation effect as an artifact of selective displaced rehearsal. Joumal of Memory \& Language, 26, 589-607.

WATKINS, M. L., \& SEChler, E. S. (1988). Generation effect with an incidental memorization procedure. Journal of Memory \& Language, 27, 537-544.

(Manuscript received November 18, 1991; revision accepted for publication May 26, 1992.) 